Literaturwissenschaft und politische Kultur 


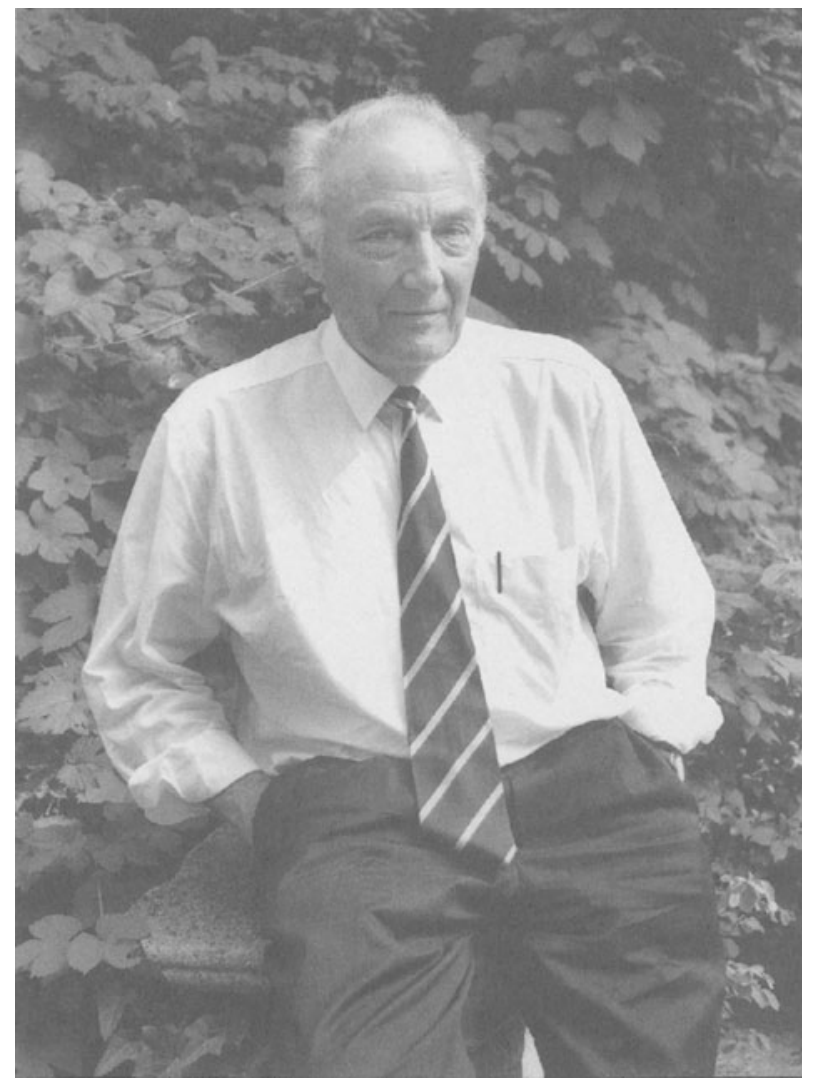

Eberhard Lämmert 


\title{
Literaturwissenschaft und politische Kultur
}

\author{
Für Eberhard Lämmert \\ zum 75. Geburtstag
}

Herausgegeben von

Winfried Menninghaus und Klaus R. Scherpe

Verlag J. B. Metzler

Stuttgart - Weimar 
Die Deutsche Bibliothek - CIP-Einheitsaufnahme

Literaturwissenschaft und politische Kultur : für Eberhard Lämmert zum 75. Geburtstag / hrsg. von Winfried Menninghaus und Klaus R. Scherpe. - Stuttgart ; Weimar : Metzler, 1999 ISBN 978-3-476-01734-5

ISBN 978-3-476-01734-5

ISBN 978-3-476-03797-8 (eBook)

DOI $10.1007 / 978-3-476-03797-8$

Dieses Werk einschließlich aller seiner Teile ist urheberrechtlich geschützt. Jede Verwertung außerhalb der engen Grenzen des Urheberrechtsgesetzes ist ohne Zustimmung des Verlages unzulässig und strafbar. Das gilt insbesondere für Vervielfältigungen, Übersetzungen, Mikroverfilmungen und die Einspeicherung und Verarbeitung in elektronischen Systemen.

Frontispiz: (C) Phoenix Agentur für Bildjournalismus, Berlin

(C) 1999 Springer-Verlag GmbH Deutschland

Ursprünglich erschienen bei $\mathbb{C}$ J.B. Metzlersche Verlagsbuchhandlung und Carl Ernst Poeschel Verlag GmbH in Stuttgart 1999 


\section{Inhalt}

Vorbemerkung der Herausgeber $\ldots \ldots \ldots \ldots \ldots \ldots$

I. Formen des Erzählens in Literatur und Kulturgeschichte

Claudia Schmölders

Josef und sein Bruder. Sigmund Freuds Volkserzählung . . . . . . . 9

Hans-Joachim Neubauer

Maria und andere UFOs, oder: Der Erzählforscher . . . . . . . . 19

GÜNTER Peters

Ein Prometheus im Schriftstellen? Lukians Gattungspoetik im Spiegel des Mythos . . . . . . . . . . . . . . 30

JiANMING ZHOU

Das Zwiegespräch zwischen Erzähler und Leser und das Vorwort der Herausgeberfiguren . . . . . . . . . . . . . . . 39

ANSELM HaVerkamp

Die Unruhen in Norwegen. Melancholie und Anamorphose in Goethes »Hamlet $\ldots \ldots \ldots \ldots \ldots$. . . . . . . . . . . 47

MarianNe SCHUlleR

Geschichte in Geschichten. Zu einer Anekdote Kleists . . . . . . . . 55

ECKART GOEBEL

$»$ Die Kuh«. Zur Kompositionskunst Friedrich Hebbels . . . . . . . 63

WALTER FäHNDERS

Die Fahrt in den Straßengraben. Narrative Strategien in Manifesten der europäischen Avantgarde . . . . . . . . . . . . . . . . 74

LOTHAR MÜLLER

Schichtende. Über Franz Fühmanns Fragment »Im Berg « . . . . . . . 83

\section{Poetik des Romans}

HartMut EgGerT

Roman und Wissenschaft im 19. Jahrhundert. Ein Essay . . . . . . 97

INKA MÜLDER-BACH

"Abreißende Anfänge«. Über Literatur und Unfall . . . . . . . . . . 107 


\section{Winfried Menninghaus}

Kafkas thanatographisches "Genießen " und Walter Benjamins RomanPoetik . . . . . . . . . . . . . . . . . . . . . . 117

Justus FetsCher

Mach keine Geschichten. Die Historie und der Trost des Erzählens in Jurek Beckers "Jakob der Lügner" . . . . . . . . . . . . . . . 126

KLAUS R. SCHERPE

Geschichten im Posthistoire. Christoph Ransmayrs Nachkriegsroman der Zweiten Generation . . . . . . . . . . . . . . . . . . . . 135

FRITZ WAHRENBURG

`Originale und Genies - Lichtenberg über Romanautoren . . . . . . 145

III. Literatur und andere Künste, Medien und diskursive Praktiken

ROBERT STOCKHAMMER

"A Proposal for correcting modern Maps «: Literatur und Kartographie

bei Jonathan Swift . . . . . . . . . . . . . . . . . . . 157

Philippe Despoix

Literarische Imagination und Naturgeschichte. Rétif de la Bretonnes

"Découverte australe" . . . . . . . . . . . . . . . . . 168

INGE BAXMANN

Verbindung der Künste und Verknüpfung der Sinne.

Zur Wagner-Rezeption der Avantgarde in Frankreich . . . . . . . . . 179

Gregor GUMPERT

"... tout moment est un berceau et un cercueil «. Augenblicks-Immanenz bei Walter Pater und Marcel Schwob . . . . . . . . . . . . . 188

Gundolf S. FREYermuth

Cyberfiktion \& Gesamtdatenwerk. Über die Adaptation der Künste an ihre Digitalisierung . . . . . . . . . . . . . . . . . . 197

Barbara NaumanN

Gesichter von Chuck Close. Ein Gespräch über Bilder . . . . . . . . 206

Ulrich Profitlich

Das Drama als »öffentliche« Gattung. Zur frühen Dramatik Martin Walsers . . . . . . . . . . . . . . . . . . . 218 
JÜRGEN SCHUTTE

Spurensicherung. Über die elektronische Edition der Notizbücher von

Peter Weiss . . . . . . . . . . . . . . . . . 226

IV. Literaturrezeption und politische Kultur

MarTin ReCTOR

Zur Anthropologie von Jakob Michael Reinhold Lenz . . . . . . . . 239

Wendula Dahle

Eine »unwürdige « Fachdidaktik? Anläßlich der Kalendergeschichte von

B. Brecht, "Die unwürdige Greisin", als Schullektüre . . . . . . . . 248

JÖRN STÜCKRATH

Anregungen zur Analyse literarischer Texte . . . . . . . . . . 258

CHRISTOPH KÖNIG

Loslösungsakte. Zur Vernunft in literarischen Werken . . . . . . . . 268

Fawzi Boubia

Exilliteratur und Entgrenzungskultur. Identität und Alterität bei Elias

Canetti . . . . . . . . . . . . . . . . . . . . . . 274

Eva Kolinsky

Wohnort Deutschland. Ansätze jüdischer Identität nach dem

Holocaust . . . . . . . . . . . . . . . . . . . . 281

RALF SCHNELL

Kunst, Geist und Macht . . . . . . . . . . . . . . . . . . . 290

Literaturverzeichnis Eberhard Lämmert . . . . . . . . . . . . . 303 


\section{Vorbemerkung}

"Das Bedeutende, das ist nichts weiter als das Beziehungsreiche " - so ein Schlüsselwort Thomas Manns, das dem Literaturwissenschaftler zugedacht sein könnte. Eberhard Lämmert nimmt es in einer seiner vielen Handreichungen für Leser und Studierende als Warnzeichen gegen die "Unterwerfung unter die exegetische Weisheit des Tonangebenden " - als Warnzeichen insbesondere gegen solche Interpreten, die Lektüre auf das Herauspräparieren von Botschaften reduzieren. Dahinter steht die Bedenklichkeit, daß man mit den zu Botschaftern erhöhten oder erniedrigten Dichtern so verfahren könne wie z. B. mit Thomas Mann, dem Lämmerts Universität, die Rheinische FriedrichWilhelms-Universität zu Bonn, unter dem Naziregime die Ehrendoktorwürde abgesprochen hatte.

Das Beziehungsreiche ist das Strukturierte. So und nicht anders läßt sich in Ausbildung und akademischem Werdegang der frühen Jahre in Bonn-Bad Godesberg Eberhard Lämmerts Einstieg in die Wissenschaft beschreiben. Die Formgebung, die anfangs noch »Gestaltung « hieß und heute auf den Namen System oder Diskurs hört, war bedeutsam und faszinierend zugleich: noch vor der Beschäftigung mit Literatur, von Hause aus und beim Studium der Bergbauwissenschaften Mineralogie und Geologie, und dann in der morphologischen Doktorandenwerkstatt Günther Müllers, welche die Bauformen des Erzählens als Meisterstück hervorbrachte. Der Strukturgedanke, das weiß später der Wissenschaftshistoriker Lämmert zu berichten, schützte vor 1945 gegen den ideologischen Mißbrauch und nach 1945 gegen die metaphysische Überhöhung der Dichtung. Die "Dichtung als Dichtung" in ihr Recht zu setzen - umgedacht und umformuliert als das Eigengewichtige der Literaturanalyse - blieb gleichwohl eine Verpflichtung. Aber nicht nur dies. Sie öffentlich und verantwortlich zur Geltung zu bringen - in der Form des Unterrichts und der Vermittlung, in ihrer wirkungsgeschichtlichen, sozialen und interdisziplinären Façon -, das wurde zur Aufgabe.

Die Wege der Literaturforschung seit den 50er Jahren ist Eberhard Lämmert nicht nur mitgegangen. Er war ein sorgsamer und entschiedener Wegbereiter. Als prägende Persönlichkeit der Wissenschaft, des Wissenschaftsbetriebs und der Hochschulpolitik konnte er die Hauptstraßen nicht verlassen, obwohl er die besten Gedanken vielleicht lieber abseits des Weges noch ein wenig weiter gepflegt hätte. Aufmerksame Leser spüren in seinen Büchern, Aufsätzen, universitätspolitischen Schriften und Erklärungen, wie das 'Mannigfaltige sein Recht fordert, bevor die 'Einheit Vereinheitlichung, die dann verpflichtet, über mehr als den eigenen Einfall und das allein Sachdienliche zu sprechen. Ein zentrales Thema wie das der zwei einander unnötig fremden Kulturen der Natur- und der Geisteswissen- 
schaften läßt sich in Lämmerts Werkstatt, unmerklich fast und behutsam angepackt, vom Rande her besichtigen. In einem Aufsatz über den Beziehungsreichtum der Wolken zum Beispiel. Die Naturerscheinung bei Alexander von Humboldt wird verglichen mit Wilhelm von Humboldts Mitteilungen zum sinnlichen Klang-Ereignis beim Aussprechen des Wortes »Wolke«. So schön und zwanglos läßt sich Aufschluß gewinnen über das Hauptsächliche.

Wissenschaft als Beruf? Max Webers Bekenntnis zu einer allein der Sache dienenden inneren Hingabe hat Eberhard Lämmert stets verbunden mit der Aufklärung über die eigene Tätigkeit, über die Germanistik als Institution und ihre Geschichte. Wie war umzugehen mit der nach dem "Zusammenbruch" andauernden Verklärung der "geistigen Führerschaft " der Dichtung und mit der Dienstbarkeit gegenüber dem Werk in der werkimmanenten Methode, die so viel andere, verschwiegene Dienstfertigkeit einschloß? Eine Antwort gab Lämmert in seiner Aufsehen erregenden Rede auf dem Münchner Germanistentag von 1966 »Germanistik - eine deutsche Wissenschaft «: ein Skandalon, das die versammelte, beim Alten verbliebene Fachdisziplin selber zu verantworten hatte. Eine andere Antwort gab er in einem Festschriftaufsatz für Richard Alewyn, den Exilierten, über einen Lieblingsdichter des "Deutschtums «, Joseph von Eichendorff, eine Arbeit über dessen »Wirkungsgeschichte «, wie Lämmert noch vor dem Aufkommen der »Rezeptionsästhetik « sagte. Hier wurde aus germanistischer Dichterverehrung Wissenschaftsgeschichte der Germanistik. Bei der Lektüre erschloß sich, wie beides zusammenhängt: das Dichterwort und die Philologenworte über den Dichter, mit denen das Dichtwerk in Anspruch genommen wurde. Auf die rebellierenden Berliner Studenten von 1967, viele von ihnen Germanisten, und auf die muffig gewordene Ordinarienuniversität reagierten Eberhard Lämmert und Peter Szondi, die jüngeren Mitglieder der Philosophischen Fakultät, mit einer bemerkenswerten Noblesse, die den älteren Kollegen damals wohl angestanden hätte. Es waren das Feingefühl und die Sorge um das Schicksal der Mannigfaltigkeit in der Vereinheitlichung der Meinungen, die ihr Handeln bestimmten: eine Mahnung an die Studenten, die blaue Blume der Romantik nicht nur rot einzufärben, und ein Entsetzen über die Fühl- und Stillosigkeit einer Fakultät und Universität, die "zu ihren eigenen Lebensfragen und zu denen der Gesellschaft mit nichts als mit den Paragraphen ihres Ordnungsrechts die Antwort suchte «. So Eberhard Lämmert in einem Aufsatz für Robert Minder, den geschätzten Kollegen im nahegelegenen Frankreich, zum 70. Geburtstag.

Die historische Erfahrung und die Einsicht, daß der Umgang mit Büchern und der Umgang mit Menschen zusammenhängen und zusammenhängen sollen - so Peter Szondis Vermächtnis -, hat Eberhard Lämmert wohl die Kraft gegeben, das zu verbinden, was andere als Einheit eher abteilen oder 
sogar verteufeln: leidenschaftliche Rede und Sachlichkeit aus Passion, Autonomie und Engagement, Eigenart und Nachbarschaft, Geistesgegenwart und Traditionsbewußtsein, Intimität des Lesens und Forschens in der Bibliothek, dem »überdachten Labyrinth «, zum einen, aber auch die Öffnung und Erweiterung der zunächst von der Liebe zum Sachlichen und Fachlichen geprägten wissenschaftlichen Tätigkeit zum anderen.

Öffnung mit der Konsequenz der Öffentlichkeit, auch zur politischen Öffentlichkeit. Eberhard Lämmert hat sie nicht gescheut, ja manchmal sich ihr geradezu ausgesetzt. Das Rektorat hat in der deutschen Universitätsgeschichte so mancher Gelehrter der Philosophischen Fakultät angestrebt und erreicht. In der Regel geschah dies im Honoratiorenstil und in Übereinkunft mit dem Zeitgeist und der Geisteshaltung der Zunft, die es zu repräsentieren galt. Als Präsident der Freien Universität galt Eberhard Lämmert vielen Kollegen als ein Abtrünniger, weil er sich für die Gruppenuniversität, für eine Kultur des Dialogs und gegen den Radikalenerlaß aussprach. Abtrünnige verfolgt die Gemeinschaft unerbittlich, wie Peter Glotz, der Partner im Senatorenamt, am Ende von Lämmerts 7jähriger erfolgreicher Amtszeit festhielt.

Erfolgreich war Lämmert für seine Institution, die Freie Universität, die er im Wissenschaftsrat, in der Deutschen Forschungsgemeinschaft und vor der Max-Planck-Gesellschaft überzeugend repräsentierte und dadurch in die scientific community zurückführte. Erfolgreich ist er seitdem durch die Autorität, die er sich durch die gelungene Verbindung von wissenschaftlichem Renommee und hochschulpolitischer Kompetenz erworben hat. Die vielen Ämter und Verantwortlichkeiten, für die er bis heute einsteht, werden überstrahlt durch die zwei wichtigsten Aufgaben der letzten zehn Jahre: die Präsidentschaft der Deutschen Schillergesellschaft, verbunden mit der Leitung des Literaturarchivs in Marbach, und die Gründung des Zentrums für Literaturforschung in Berlin-Mitte in der Nachfolge der ehemaligen DDR-Akademie. Die Aufbauleistungen hier wie dort werden als kulturpolitische Leistungen ersten Ranges gewürdigt. Lämmert mag hierin die zumindest teilweise Erfüllung eines lange gehegten Wunschtraums sehen: die Errichtung eines Max-Planck-Instituts für die Literaturwissenschaft. Darüber steht nur noch das Persönliche: die Fähigkeit zum Miteinander und zum Dialog, die »rettende Liebe ", wie Theodor Lessing einmal gesagt hat.

Öffnung und Erweiterung mit Konsequenz auch in der Wissenschaft selber. Interdisziplinarität hat Lämmert nicht nur gefordert, sondern verwirklicht, Schritt für Schritt. Bevor ihn, neben der ideologiekritischen Aufklärung, die Beschäftigung mit den Nachbarliteraturen und die methodische Zusammenarbeit mit den Nachbardisziplinen an der germanistischen Bastion der 'Einheit des Faches` zweifeln ließ, hat er die angestammte Verpflichtung erfüllt und sich als Neugermanist im altgermanistischen Fach mit einer Arbeit über 
die Teichnerreden in Bonn habilitiert. Daher rührt vielleicht auch die ständig erneuerte Aufforderung an sich selber und an andere, die gewünschten Veränderungen in Forschung und Lehre mit dem eigenen Vermögen sorgfältig vorzubereiten und möglichst unprätentiös zu handhaben. Dabei betraf die Veränderung der Inhalte und der Mittel ihrer Handhabung nichts geringeres als die Erweiterung und die Neudefinition des Literaturbegriffs selber, die veränderte öffentliche Rolle des Schriftstellers und Literatur-Intellektuellen, die als `Literatursoziologie< zusammengefaßten "Nachbarschaften " der Literatur im Literaturbetrieb - vom Verlagswesen bis zum Literaturunterricht und das in Deutschland wenig produktive Verhältnis von Literaturwissenschaft und Literaturkritik, nicht zuletzt die Rückführung der vergessenen und verfemten Literatur in das Gebäude der Literaturgeschichte. Auf all' diesen Arbeitsfeldern haben Eberhard Lämmerts Forschungen nicht nur Spuren hinterlassen, sondern prägend gewirkt.

Am eindrucksvollsten und nachhaltigsten geschah dies wohl auf seinem angestammten Gebiet der Erzählforschung von der Bonner Dissertation über die Editionen zur Romantheorie seit dem 18. Jahrhundert bis zum DFGKolloquium zur Erzählforschung von 1980. Hier vor allem, in der Spannweite vom mündlichen Erzählen bis zum Hypertext, hatte sich das zu beweisen, was unter Lämmerts kritischem Blick das ,Literarischer überhaupt aus- und haltbar macht: die Fähigkeit zum fiktionalen und poetischen Experiment, zum Durchspielen und Ausspielen des Gesellschaftlichen in seiner zwangsläufig pragmatischen und durchweg sozialen Form. Und dabei haben auch die 'Literaturverhältnisse`, wie man so unschön sagt, für ihn stets etwas Praktisches und Persönliches: im Umgang mit seinen eigenen Verlegern zum einen und zum andern durch die gute Bekanntschaft mit Schriftstellern, Uwe Johnson zum Beispiel oder Günter Grass.

Das Nachdenken über das Verhältnis der Literatur zu anderen Künsten, zur Literatur in ihrer eigenen Medialität und zur Literaturwissenschaft, verstanden als Kulturwissenschaft (im Rückgriff und im Vergleich zu der von Jacob Grimm geleiteten Gelehrtenversammlung der Germanisten von 1846), hat Eberhard Lämmert wie selbstverständlich, wenngleich verbunden mit einigen institutionellen Anstrengungen, zur Allgemeinen und Vergleichenden Literaturwissenschaft gebracht. »Vergleichend ermittelte Eigenschaften literarischer Texte nach allgemeinen Kategorien zu ordnen und auch zu deuten «: Das war für Lämmert schon das Projekt der Aristotelischen Poetik, ebenso wie "für die gebildeten Literaturkenner, auch unter den Gelehrten, ein abendländischer und später ein weltliterarischer Horizont vielfach selbstverständlich « war. Als universitäre Disziplin jedoch mußte sich die Allgemeine und Vergleichende Literaturwissenschaft erst gegen die nationalen Begrenzungen der Einzelphilologien durchsetzen, und Lämmert hat als Historiker dieser 
jungen Disziplin ihre engen Beziehungen zu historischen Katastrophen und epochalen Umwälzungen betont: »Es ist denkwürdig genug, daß erst der Kriegs- und Revolutionsaufruhr und sodann die blutigen Vertreibungen des 20. Jahrhunderts zu einer international verstrebten, allgemein und vergleichend arbeitenden Literaturwissenschaft den Grund gelegt haben."

Als Nachfolger Peter Szondis am Seminar für Allgemeine und Vergleichende Literaturwissenschaft der Freien Universität - dem Seminar, zu dessen Initiatoren und Begründern er mehr als ein Jahrzehnt zuvor gehört hatte blieb er dem besonderen Akzent auf der allgemeinen Literaturwissenschaft verpflichtet, durch den schon Szondi das Berliner Institut von anderen, im engeren Sinne komparatistischen Seminaren markant unterschieden hatte. Deutlich ausgebaut hat er zugleich die Künste-Komparatistik und Fragestellungen im Grenzgebiet von Historiographie und Literaturtheorie. Noch zu Szondis Lebzeiten und vollends im längeren >Interregnum zwischen seinem Tod und Lämmerts Neuberufung war das Institut zu einem Ort intensiver Beschäftigung und Weiterentwicklung poststrukturalistischer Theorien und Praktiken des Lesens geworden. Szondi selbst hatte diese Entwicklung sowohl durch seine späteren Schriften als auch durch Gasteinladungen Jacques Derridas und Paul de Mans entschieden begünstigt, behielt aber gleichwohl gewisse Reserven. Lämmert hat die Virulenz und Produktivkraft dieses Denkens gelten und weiter wirken lassen, gleichwohl aber selbst stets dezidiert am hermeneutischen "Besteck « seiner Disziplin und an der Notwendigkeit erkenntnisleitender Sinnentwürfe festgehalten. Auch wenn gelegentlich milde ironische Töne für die »Auslegungs-Vergnügungen « der Sinn-Dekonstruierer, der ssubversiven « Unabschließbarkeits- und Unentscheidbarkeitstheoretiker gefunden wurden, blieb er doch stets ein Mentor für viele, die nicht durchweg seine wissenschaftlichen Positionen teilten. Unter seiner Ägide konnte eine breit gestreute Vielfalt von Wissenschaftlern gedeihen. Durch diese Generösität des Lassens hat Eberhard Lämmert das Berliner Institut für Allgemeine und Vergleichende Literaturwissenschaft ebenso geprägt wie durch den sanften Nachdruck seiner Argumente und die unaufdringliche Autorität seines Auftretens. Er hat über viele Jahre eine einzigartige Atmosphäre selbständigen wissenschaftlichen Arbeitens gefördert, die von Urbanität, Internationalität und einer in ihrer Selbstverständlichkeit unübertroffenen Liberalität geprägt war; illiberal war er allenfalls gegen kleinliches Denkens, >Dummheit` und Machtarroganz aller Spielarten. Wer das eigentümliche Glücksgefühl erlebt hat, im Raum solcher unwahrscheinlichen sozialen und intellektuellen Tugenden zu arbeiten, für den ist es nicht länger ein nur historisches Datum, daß im 18. Jahrhundert die Literatur und teilweise auch ihre Kritik "schöne Wissenschaften " genannt wurden.

Am Ende schlägt das zu Buche, was auch das Zustandekommen dieser 
Festgabe gefördert hat, die seine ehemaligen Studentinnen und Studenten, Promovendinnen und Promovenden, Mitarbeiterinnen und Mitarbeiter, Habilitandinnen und Habilitanden aus verschiedenen Regionen und Ländern und aus mancherlei Fachinteresse ihm zugedacht haben: der Wunsch und das Vermögen, wie Eberhard Lämmert einmal listig und weise gesagt hat, »unbeschadet aneinander vorbeizukommen «. Das klingt nach wenig und ist doch unerhört viel: im Stile des Meisters der Einspruch gegen jederlei Aufgeregtheit im Schreiben wie im Handeln, das Geschenk einer Freiheit der Wissenschaft, die souverän mit Freunden und Gegnern umgeht und im Vergangenen, trotz alledem, die Hoffnung sieht für die Zukunft, wie es der Freund und Kollege Peter Szondi formuliert hat.

Hartmut Eggert, Gert Mattenklott und Ulrich Profitlich haben an der Vorbereitung dieser Festgabe mitgewirkt, Henning Dahl-Arnold hat mit viel Geschick, Geduld und Hartnäckigkeit die Organisation des ganzen Vorhabens und die fristgerechte redaktionelle Einrichtung des Manuskripts besorgt. Bernd Lutz hat den Herausgebern durch ein großzügiges Verlagsangebot des Metzler Verlags alle Finanzierungsprobleme abgenommen und auch von der ersten Konzeption bis zur Endkorrektur stets selbst, rasch und einfallsreich für das Zustandekommen des vorliegenden Bandes Sorge getragen. Im Namen der darin durch ihre Beiträge versammelten ehemaligen Schülerschar und heutigen Kollegenschaft, aber sicher auch im Namen aller derjenigen, denen er bis heute durch seine Autorität, sein Können und seine Zuwendung hilfreich zur Seite steht, bedanken wir uns bei Eberhard Lämmert. 